\title{
Interação medicamentosa em pacientes idosos com dislipidemias em Unidade Básica de Saúde de Santana do Cariri, CE, Brasil
}

\author{
Drug-drug interaction in elderly patients with dyslipidemia \\ desease in a Basic Health Unity in Santana of Cariri, CE, Brazil
}

Recebido em: $18 / 02 / 2020$ Aceito em: $20 / 06 / 2020$
Lucilene Galdino de BARROS; Felisberto Farias SANTOS; Dárcio Luiz de SOUSA JÚNIOR; Ana Emília Formiga MARQUES Faculdade de Medicina Estácio de Juazeiro do Norte. R. Ten. Raimundo Rocha, 515, Cidade Universitária, CEP 63040-360. Juazeiro do Norte, CE, Brasil. E-mail:darciolsjr@gmail.com

\section{ABSTRACT}

This study aimed to perform an analysis of drug interactions based on data from medical prescriptions for elderly patients using drugs for dyslipidemia in a basic health unit in the city of Santana do Cariri, CE. Data collection and analysis were carried out between August and September 2018. This is a descriptive, quantitative, retrospective, and cross-sectional study. Micromedex ${ }^{\circledR}$ platform was used to identify possible drug interactions. Elderly patients with some dyslipidemia, with a minimum age of 60 and a maximum of 89 years, were selected. A total sample of 40 prescriptions was obtained, where the population was 103 prescriptions, in which $80 \%$ were female and $20 \%$ male. A total of 37 interactions was identified; among them, the drug simvastatin was more frequently interacting with other drugs, the most frequent was amlodipine. These two drugs interaction is considered serious, due to the risk of myopathies and rhabdomyolysis. In view of these results, the importance of implementing a clinical pharmacy service is observed, in order to identify possible problems related to drug-drug interactions, improving the patient pharmacotherapy and quality of life, as well as minimizing the risks for their health.

Keywords: drug-drug interaction; pharmaceutical care; dyslipidemia.

\section{RESUMO}

Este estudo teve como objetivo realizar uma análise de possiveis interações medicamentosas baseadas em dados de prescrições médicas para pacientes idosos que fizessem uso de medicamentos para dislipidemias em unidade básica de saúde na cidade de Santana do Cariri, CE. A coleta e análise dos dados ocorreu entre agosto e setembro de 2018 no município de Santana do Cariri, CE. Trata-se de um estudo descritivo, quantitativo, retrospectivo e transversal. Para a identificação das possíveis interações medicamentosas, foi utilizada a plataforma Micromedex ${ }^{\circledR}$. Foram selecionados pacientes idosos portadores de alguma dislipidemia, com idade mínima de 60 e máxima de 89 anos. A amostra total foi 40 prescrições, onde a população foi de 103 receituários, em que $80 \%$ era do sexo feminino e $20 \%$ masculine. Foi identificado um total de 37 inte- 
rações; dentre estas, sinvastatina é o que aparece com maior frequência interagindo com outros fármacos, dentre os quais, o mais frequente foi com o anlodipino. A interação desses dois fármacos provocam interação no paciente é considerada grave, pois o indivíduo corre o risco de apresentar miopatias e rabdomiólise. Diante desses resultados, é importante a implantação de um serviço de farmácia clínica, que possibilita identificar possíveis problemas relacionados a medicamentos e interações, melhorando a farmacoterapia e a qualidade de vida do paciente, beneficiando com uma assistência eficiente para minimizar riscos à saúde.

Palavras-chaves: dislipidemias; interação medicamentosa; cuidados farmacêuticos.

\section{INTRODUÇÃO}

A dislipidemia é um distúrbio sanguíneo caracterizado pelos níveis elevados de lipídios ou lipoproteínas no sangue; pode ser primária, relacionada a dieta e distúrbios genéticos, ou secundária, uma combinação de condições como diabetes mellitus, alcoolismo, síndrome nefrótica, insuficiência renal crônica, hipotireoidismo, doença hepática e administração de fármacos (1). A dislipidemia pode ocorrer de forma incipiente desde a infância; no entanto, o desenvolvimento acontece com mais frequência na vida adulta, dependendo de fatores como os hábitos alimentares e o estilo de vida de cada ser humano (2).

O tratamento farmacológico pode ser feito com: resinas de ácidos biliares (colestiramina, colestipol, colessevelam), niacina, inibidores da HMG-CoAredutase (atovarstatina, fluvastatina, lovastatina, pravastatina, rosuvastatinae sinvastatina), fibratos (genfibrozila, fenofibrato, clofibrato), ezetimiba e suplementação com óleo de peixe. $\mathrm{O}$ mecanismo de ação das estatinas envolve inibição competitiva da HMG-CoA-redutase, formando posteriormente o colesterol. A inibição da síntese endógena leva à supra-regulação da síntese dos receptores de LDL, ou seja, aumenta a quantidade de hepatócitos na superfície dos receptores, resultando em uma maior remoção de LDL do plasma (3).

Para o tratamento das dislipidemias podem ser utilizados fármacos (tratamento farmacológico), mas pode também ser recomendado mudanças no estilo de vida, bem como nos hábitos alimentares. As estatinas são hoje os fármacos de primeira escolha, e que reduzem, em média, os valores de LDL-c entre 10 a $25 \%$, diminuindo os eventos cardíacos e aumentando qualidade de vida dos indivíduos. Os hipolipemiantes, além de reduzirem os níveis de colesterol, devem reduzir os riscos $(4,5,6)$.

Para os medicamentos prescritos que apresentam um estreito índice terapêutico há mais chances de ocorrer interação medicamentosa (IM); principalmente aqueles cjo mecanismo envolve o citocromo CIP450. A quantidade de medicamentos prescritos é muito importante, pois o uso concomitante de vários fármacos pode causar entre 3\% a $5 \%$ de interação em pacientes que utilizam uma grande quantidade desses medicamentos $(7,8)$.

A interação medicamentosa pode ser classificada como um evento clínico, ocorrido quando a toxicidade ou efeito de um fármaco é interligado à presença de outras substâncias. A modificação de um medicamento pelo contato com outro fármaco, bebida, alimento ou ainda certos agentes químicos ou ambiental, é chamada de interação medicamentosa. Essas interações, em alguns casos, podem ser confundidas com uma reação adversa, resultado da doença e não pela interação entre fármacos $(9,10)$.

Quanto à interação provocada pelo o uso das estatinas várias anomalias metabólicas podem ocorrer, miopatias, toxicidade musculares, que podem ser elevadas ou não. As estatinas em doses altas podem aumentar o risco de sintomas musculares, pois eleva a lipofilia, as interações dos medicamentos e o metabolismo via citocromo P450 (11).

Eventos com tais sintomas são mais prejudiciais em casos de pessoas em cuidados intensivos, pós-cirúrgicas e principalmente na população idosa. Este último caso tem se tornado de extrema importância por poderem apresentar saúde debilitada em decorrência do envelhecimento. Estima-se que mais de $50 \%$ dos idosos podem sofrer devido a 
alguma interação medicamentosa $(12,13)$. No entanto, a intervenção farmacêutica é uma importante estratégia de melhoria na farmacoterapia; garante qualidade e segurança para o idoso, diminui os impactos dos riscos de saúde, abrange uma reeducação em saúde (14).

Desta forma, a Farmácia Clínica pode prevenir e ajudar a solucionar possíveis falhas envolvidas na farmacoterapia em doenças crônicas que acometem principalmente os idosos (17). O acompanhamento farmacoterapêutico de idosos com dislipidemias tem auxiliado na prevenção de episódios cardiovasculares, aterosclerose e na prevenção de doença arterial coronariana (16).

O objetivo deste trabalho foi fazer uma análise de possíveis interações medicamentosas, baseadas em dados das prescrições médicas de pacientes idosos que fizeram uso de medicamentos para dislipidemias, em unidade básica de saúde no período de agosto a setembro de 2018 no município de Santana do Cariri, CE.

\section{MÉTODO}

Trata-se de um estudo descritivo, quantitativo e transversal realizado na Unidade Básica de Saúde, da Mini Maternidade Rosa Siqueira Coelho no distrito de Dom Leme da cidade de Santana do Cariri, no Estado do Ceará, que está situada na microrregião do Cariri, ocupando uma área de $855,563 \mathrm{~km}^{2}$, com população estimada em 17.489 habitantes (4).

A coleta e a análise dos dados foram feitas no período de agosto a setembro de 2018, para caracterizar interações medicamentosas em prescrições de pacientes idosos portadores de algumas dislipidemias que foram obtidos através dos prontuários tendo como critérios de inclusão a idade mínima de 60 a 89 anos.

Foram excluídos pacientes com menos de 60 anos que utilizassem menos de três medicamentos. Na busca sobre interações medicamentosas foi utilizada a plataforma da Micromedex ${ }^{\circledR}(17)$, classificando-as como leve, moderada e grave e com documentação boa e excelente.

O trabalho teve aprovação do comitê de ética em pesquisa sob o número do parecer 2.868.318, que será anexado no apêndice. Os dados obtidos na pesquisa foram transferidos para Software Microsoft Office Excel 2010.

\section{RESULTADOS E DISCUSSÃO}

A população do estudo foi constituída por 40 prescrições de idosos, sendo $32(80 \%)$ do sexo feminino e $08(20 \%)$ do sexo masculino. Todos utilizavam um hipolipemiante para tratamento de hipercolesterolemia, além de outros medicamentos de uso contínuo. A distribuição desses pacientes, quanto à idade, é mostrada na Figura 1.

Figura 1. Distribuição, por idade, dos pacientes apresentando dislipidemia, atendidos em Unidade Básica de Saúde, de Santana do Cariri, CE (agosto-setembro de 2018).

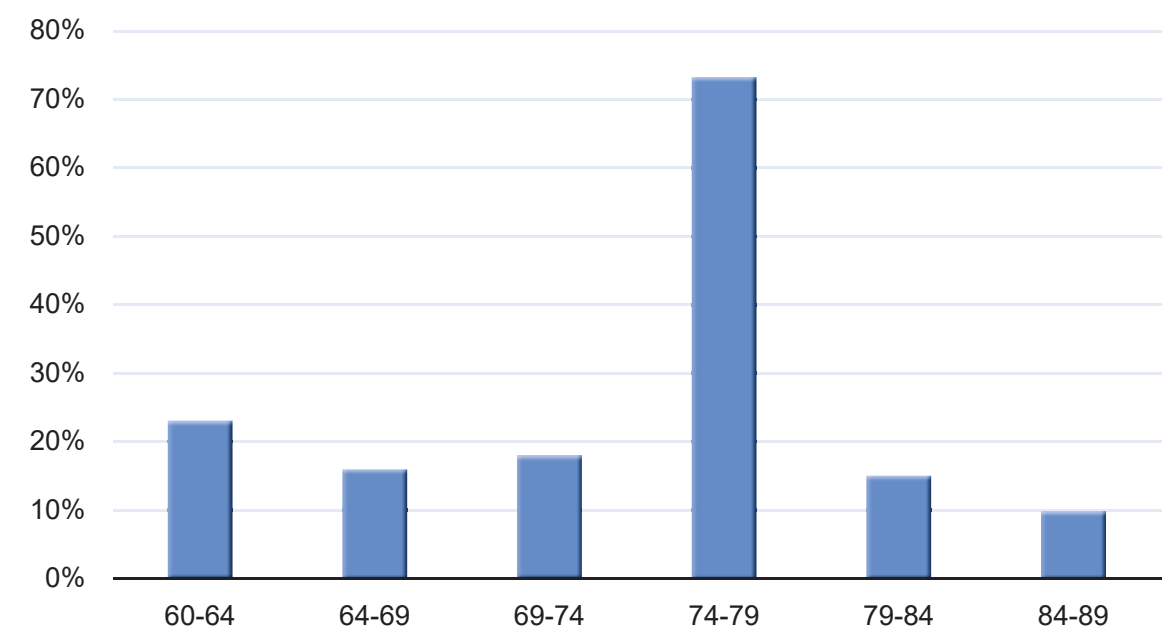


Com relação ao número de fármacos prescritos por paciente, foram 43 medicamentos para os 40 receituários do estudo, e que cada prescrição tinha, no mínimo, 4 medicamentos, e alguns pacientes chegavam utilizar 8 tipos diferentes de medicamentos. Todos os pacientes, além da dislipidemia, apresentavam outras doenças crônicas como hipertensão, diabetes mellitus ou gastrites. Quanto aos medicamentos, aqueles correlacionados com a patologias mais prevalentes foram sinvastatina, hidroclorotiazida, metformina, anlodipino e ácido acetilsalicílico (AAS, $100 \mathrm{mg}$ ). Na Tabela 1 foram expostos os fármacos, as frequências em que aparecem e a porcentagem e a enfermidades de acordo os resultados obtidos.

Ao comparar idade com o número de medicamentos utilizados, a população com mais de 74 anos fazia uso de mais medicamentos com relação aos demais. Isso pode ser justificado porque com o avançar da idade, outras comorbidades vão surgindo (26).

A partir da plataforma Micromedex ${ }^{\circledR}$ (17) foram determinados os tipos de risco da interação (Leve, Moderado ou Grave). Para isso foi usada documentação científica considerada Excelente (se estudos controlados direcionavam a existência da interação medicamentosa); Boa (se a documentação comprovava que houve a interação, mas faltavam estudos bem controlados); Regular (se a documentação disponível era escassa, mas as bases farmacológicas permitiam suspeitar da interação); Pobre (quando a documentação estava limitada a estudos de casos); Improvável (quando a documentação era pobre e faltavam bases farmacológicas); e Desconhecida (quando a documentação sobre a interação não era conhecida).

A partir dessa classificação as interações foram definidas como Gravíssima ou contra-indicada (quando o uso concomitante com outro medicamento não era indicado); Grave (se a interação oferecesse riscos à vida do paciente e precisasse de uma intervenção médica de urgência); Moderada (quando a interação poderia resultar em exacerbação da condição clínica do paciente ou demandar alteração da terapia); Leve (quando a interação poderia ter efeitos clínicos limitados, sem demandar alterações na terapia medicamentosa); e Desconhecida (quando não havia definição da gravidade).

A amplitude de interações variou de 0 a 37. Destas, 35,1\% foram classificadas como graves, $56,7 \%$ como moderadas e $8,1 \%$ como leves. O principal medicamento envolvido nas interações potenciais foi o Anlodipino, presente em 12 interações, o que corresponde a $32,4 \%$ do total. As interações classificadas como graves, correspondia à associação entre o bloqueador de canal de cálcio anlodipino e o hipolipemiante sinvastatina. A Tabela 2 mostra as principais interações com sinvastatina encontradas.

Tabela 2. Principais interações medicamentosas envolvendo sinvastatina encontradas nas prescrições para pacientes apresentando dislipidemia, atendidos em Unidade Básica de Saúde, de Santana do Cariri, CE (agosto-setembro de 2018).

\begin{tabular}{|l|c|l|l|}
\hline Interação com & Frequência & \multicolumn{1}{|c|}{ Riscos } & \multicolumn{1}{c|}{ Consequência } \\
\hline Anlodipino & 12 & Grave, documentação Boa & Risco de miopatia e rabdomiólise. \\
\hline Glibenclamida & 11 & Moderado, documentação Boa & $\begin{array}{l}\text { Aumenta a concentração plasmática do } \\
\text { hipoglicemiante. }\end{array}$ \\
\hline Omeprazol & 07 & Moderado, documentação Excelente & Aumenta a concentração sérica da estatina. \\
\hline Risperidona & 01 & Grave, documentação Boa & $\begin{array}{l}\text { Aumenta concentração sérica da estatina e ocorre } \\
\text { risco de miopatia e rabdomiólise. }\end{array}$ \\
\hline Carbamazepina & 02 & Leve, documentação Boa & Reduz o efeito das estatinas. \\
\hline Digoxina & 02 & Moderado, documentação Boa & Aumento dos níveis plasmáticos da digoxina. \\
\hline Ciprofibrato & 01 & Contraindicado, documentação Excelente & Aumenta risco de ocorrer miopatia e rabdomiólise. \\
\hline Levotiroxina & 01 & Leve, documentação Boa & $\begin{array}{l}\text { Ouso de sinvastatina c/ levotiroxina, pode resultar } \\
\text { na diminuição da eficácia da levotiroxina. }\end{array}$ \\
\hline
\end{tabular}


O fato de as mulheres se cuidarem mais que os homens e procurarem mais os serviços de saúde para prevenção, resulta em crescente número de mulheres envelhecendo com mais saúde, com maior longevidade em relação aos homens. Apresentam menor exposição a determinados de risco a saúde, estando relacionado ao estilo de vida, tais como uso abusivo de álcool e tabagismo em menor prevalência, bem como diferenças quanto à atitude em relação a doenças e incapacidades (18).

Estudos feitos por Sullivan e cols (2004) mostraram que a prevalência de hipercolesterolemia é maior em idosos do sexo feminino (42\%), enquanto em homens é de $25 \%$ aproximadamente A taxa se mantém acima de $240 \mathrm{mg} / \mathrm{dL}$ e vai caindo com o avançar da idade (19). A hipertensão está associada a distúrbios metabólicos, que pode estar agravar e alterar o funcionamento de órgãos pela presença de outros fatores de risco, como dislipidemia, obesidade abdominal, intolerância à glicose e Diabete mellitus (20). Em geral idosos utilizam, em média, entre três a oito medicamentos por dia, o que pode ocorrer em alguma possibilidade de uso indevido, levando a ocasionar problemas ou possíveis complicações para tais pacientes (21).

Oliveira e cols (2016), destacam que o componente básico da assistência farmacêutica tem uma relação muito próxima com as demandas dos serviços terapêuticos medicamentosos no âmbito do SUS, pois correspondem aos principais agravos de saúde na atenção básica que acometem os idosos, tais como a HAS, Diabetes mellitus e Dislipidemias (22).

A interação de anlodipino e sinvastatina, por meio de doses elevadas, comumente em idoso, apresenta risco para a saúde do paciente pelo fato de anlodipino aumentar a exposição da sinvastatina aos seus metabólitos, aumentando o risco do paciente desenvolver miopatias, dentre elas a rabdomiólise, uma necrose muscular resultando em mialgia e/ou liberação de enzimas musculares na circulação (23).

Para Brunton e cols (2012), em pacientes que realizam tratamento para hipotireoidismo, os níveis de hormônio T4 tireoidiano estão reduzidos; com isso ocorre um aumento da concentração plasmática de LDL, pois o T4 estimula a síntese de receptores hepáticos de LDL. Dessa forma, o uso de sinvasta- tina pode influenciar diminuindo o efeito do hormônio tireoidiano, devendo haver um acompanhamento especial da equipe multidisciplinar para esses pacientes idosos com tais doenças crônicas (7).

De acordo com a plataforma Micromedex ${ }^{\circledR}$ (17) o ciprofibrato não deve ser utilizado com a sinvastatina, pois além de ser contraindicado por duplicidade, aumenta risco de miopatias e rabdomiólise. No uso da sinvastatina com a glibenclamida (11, $29,7 \%$ ) ocorre risco moderado podendo aumentar a concentração plasmática do hipoglicemiante.

Outro medicamento prevalente foi o omeprazol $(7,18,9 \%)$, utilizado por 40 pacientes para prevenir gastrite e dores estomacais. O uso do omeprazol com sinvastatina reduz o efeito da estatina.

A sinvastatina, apesar de ser considerado um fármaco seguro e bem tolerado, pode levar a inúmeras interações quando associada a outros fármacos como amiodarona, anlodipino, carbamazepina, ciprofibrato, risperidona e diltiazem, que podem ser consideradas graves, com risco de efeitos colaterais, como miopatias, moderadas temos glibenclamida, digoxina e omeprazol (24).

Os resultados encontrados nesse trabalho condizem com análise de Lutz e cols (2017) que destacaram que as alterações fisiológicas oriundas do processo de envelhecimento afetam de maneira considerável a farmacocinética e farmacodinâmica dos medicamentos em idosos e que a polifarmácia é fator condicionante ao aumento da incidência de interações medicamentosas (25), bem como as reações adversas a medicamentos (RAM) (26).

Dessa forma, esse estudo condiz com resultados encontrados por Farias e cols (2019) que definiram a polifarmácia como diversas prescrições recebidas por consultas médicas de especialidades diferentes, sem a necessária avaliação do risco-benefício do uso desses medicamentos e sem relacionar com os medicamentos que o paciente já utiliza, podendo agravar os casos de interações medicamentosas (27).

\section{CONCLUSÃO}

Foram detectados 37 tipos de interações medicamentosas da sinvastatina com outros fármacos prescritos para várias doenças crônicas não transmissíveis. A presença de uma equipe multidiscipli- 
nar, na qual se insere o farmacêutico é um componente indispensável na avaliação da farmacoterapia pois, ainda que a polifarmacoterapia seja, em alguns casos justificada por evidencias clínicas ou protocolos terapêuticos, há de se avaliar os riscos/ benéficos da prescrição.

Dentre as interações encontradas, algumas não constituiam risco grave ao paciente, não sendo ne- cessário interromper o tratamento. Contudo, devem ser monitoradas e acompanhadas pela equipe básica de saúde multidisciplinar. A participação do farmacêutico no acompanhamento farmacoterapêutico é indispensável no tratamento dessas patologias, pois pode contribuir de maneira significativa para a promoção e recuperação da saúde, bem-estar e qualidade de vida desses pacientes idosos.
1. Rang HP, Dalle M, Ritter JM. Farmacologia, $7^{\mathrm{a}}$ edição, Elsevier, 2011.

2. Faria EC, Dalpino FBT, Takata, R. Lipídeos e lipoproteínas séricos em crianças e adolescentes ambulatórias de um hospital universitário público. Rev Paul Ped. 2008;26(1):54-58. DOI: 10.1590/S010305822008000100009 .

3. Wells BG, Dipiro JT, Schwinghamer TL, Dipiro CV. Manual de Farmacoterapia. 2a ed. 2016.

4. BRASIL. Relação nacional de medicamentos essenciais - rename 2017. Brasília: Ministério da Saúde, 2017. Disponível em: https://bvsms.saude.gov.br/bvs/publicacoes/relacao_nacional_medicamentos_rename_2017. pdf. Acesso em: 23 de outubro 2018.

5. Raal FJ, Pilcher GJ, Panz VR, Van Deventer HE, Brice BC, Blom DJ, Marais AD. Reduction in mortality in subjects with homozygous familial hypercholesterolemia associate ed with advances in lipid-lowering therapy. Circulation. 2011;124(20):2202-2207. DOI: 1161/ CIRCULATIONAHA.111.042523

6. Katzung BG. Farmacologia Básica e Clínica. Editora Guanabara Koogan, 10. Ed; 2010.

7. Brunton LL, Chabner BA, Knollman BC. Goodman \& Gilman as bases farmacológicas da terapêutica. 12 ed. Rio de Janeiro: McGraw Hill; 2012.

8. Reis LA, Torres GV, Xavier TT, Silva RAR, Costa IKF, Menders FRP. Percepção do suporte familiar em idosos de baixa renda e fatores associados. Texto ContextoEnferm. 2011;20:52-58. DOI: 10.1590/S0104-07072011 000500006

9. Caccia S, Pasina L, Nobili A. Howpre-marketing data canbeused for predicting the weight of drug interactions in clinical practice. Euro J Internal Med. 2013;24(3): 217-221. DOI: 10.1016/j.ejim.2012.12.006

10. BRASIL.. Uso Racional de Medicamentos: Temas Selecionados. Brasília: Ministerio da Saúde. Secretaria de Ciência, Tecnologia e Insumos Estratégicos; 2012. Disponível em: http://bvsms.saude.gov.br/bvs/publicacoes/ uso_racional_medicamentos_temas_selecionados.pdf. Data do acesso: 21 de outubro de 2018.

11. Vladutiu GD, Simmons Z, Isackson PJ, Tarnopolsky M, Peltier WL, Barboi AC, Sripathi N, Wortmann RL, Phillips PS. Genetic risk factors associated with lipid lowering drug induced myopathies. Muscle \& Nerve: Offic J Amer Assoc Electrodiag Med. 2006;34(2):153162. DOI: $10.1002 /$ mus.20567

12. Faria ALG, Fais FLB, Ribeiro JM, Marialva RLH, Costa VD, Matsutani GC. Avaliação das interações medicamentosas e possíveis efeitos colaterais em pacientes idosos da clínica cardiovascular. Diál Interdiscip. 2019; 8(10):21-28.

13. Cavalcante MLSN, Alcântara RKLD, Oliveira ICLD, Aires SF, Girão ALA, Carvalho REFLD. Segurança medicamentosa em idosos institucionalizados: potenciais interações. Esc Anna Nery. 2020;24(1).

14. Oliveira LCFD, Assis MMA, Barboni AR. Assistência farmacêutica no Sistema Único de Saúde: da Política Nacional de Medicamentos à atenção básica à saúde. Ciênc e Saúde Colet. 2010;15:3561-3567. DOI: 10.1590/ S1413-81232010000900031

15. Pereira LP, Sichieri R, Segri NJ, Silva RMVG, Ferreira MG. Dislipidemia autorreferida na Região Centro-Oeste do Brasil: Prevalência e Fatores Associados. Rev Ciênc em Saúde Colet. 2015;20(6):1815-24. DOI: 10.1590/1413-81232015206.16312014

16. Xavier HT, Izar MC, Faria Neto JR, Assad MH, Rocha VZ, Sposito AC, Faludi AAV. Diretriz brasileira de dislipidemias e prevenção da aterosclerose. Arq Bras Cardiol. 2013;101(4):1-20. DOI: 10.5935/abc.2013S010

17. Micromedex. USP. MICROMEDEX 2.0: DI® Drug Information for the Health Care Professional. 2018. Disponível em: http://psbe.ufrn.br/index.php?option= com_content\&view $=$ article\&id=36\&Itemid $=248>$

18. Mauren SS, Mazo GZ, Lopes MA, Benedetti TB. Atividade Física e o Idoso: Concepção Gerontológica. 3. ed. Revisada e ampliada. Porto Alegre; 2011. 
19. Sullivan LM, Massaro JM, D’Agostino SR, Ralph B. Presentation of multivariate data for clinical use: The Framingham Study risk score functions. Statist in Med. 2004;23(10):1631-1660. DOI: /10.1002/sim.1742

20. Malachias MVB, Póvoa RMS, Nogueira AR, Souza D, Costa LS, Magalhães ME. $7^{a}$ Diretriz Brasileira de Hipertensão Arterial. Arq Bras Cardiol. 2016;107(3):14-17. DOI: $10.5935 / \mathrm{abc} .20160153$

21. Vrdoljak D, Borovac JA. Medication in the elderlyconsiderations and therapy prescription guidelines. Acta Med Acad. 2015;44(2):159-168. DOI: 10.5644/ ama2006-124.142

22. Oliveira REM, Nascimento MMG; Pereira ML. Uso de medicamentos por idosos de uma unidade de atenção primária à saúde. Rev Bras Far Hosp Serv de Saúde. 2016;7(3):30-34.

23. Passarelli MCG, Jacob Filho W, Figueras A. Adverse drug reactions in an elderly hospitalised population. Drugs Aging. 2005;22(9):767-777. DOI: $10.2165 / 0000$ 2512-200522090-00005.
24. Costa AM, Tasca SR, Apolinario APA, Borghi L. Caracterização do uso de sinvastatina pelos pacientes da terceira idade. Rev Cient Ágape. 2018;1(1):13-13.

25. Lutz BH, Miranda VIA, Bertoldi AD. Inadequação do uso de medicamentos entre idosos em Pelotas, RS. Rev de Saúde Públ. 2017;51:1-12. DOI: 10.1590/S1518-8787. 2017051006556.

26. Becerra RGC, Ríosa EV, Rodrigueza LG, Dazaa VER, Gonzálera LM. Estado de salud en el adulto mayor em atención primaria a partir de una valoración geriátrica integral. Aten Prim. 2015;47(6):329-335. DOI: 10.1016/ j.aprim.2014.07.007.

27. Faria, ALG., Fais, FLB., Ribeiro, JM., Marialva, RLH., Costa, V.D., Matsutani, GC. Avaliação das interações medicamentosas e possíveis efeitos colaterais em pacientes idosos da clínica cardiovascular. Diál Interdiscipl. 2019;8(10), 21-28. 